\title{
Leakage Modeling and Flat Field Correction Algorithm for CMOS Hyper Spectral Imaging Sensor
}

\author{
Shuyang Liu, Pradip Mainali, Klaas Tackand, and Rudy Lauwereins
}

\begin{abstract}
In hyper spectral imaging applications, the light power reaching the image sensofr is greatly reduced compared to broadband color image sensors. Consequently, some typical algorithms, such as Flat Field Correction (FFC), are not guaranteed anymore to work in the same way as in their normal operation for broadband color imaging. This is caused by transistor leakage which cannot be neglected anymore. In this paper, we propose a mathematical leakage model based on the basic transistor theory to tag the validity of the sensor response. The model has been validated by comparing the simulation results to measurements of the pixel response of a hyper spectral imager. We also demonstrate that this leakage model is able to select the proper training set for a typical FFC algorithm.
\end{abstract}

Index Terms-Leakage model, transistor leakage, CMOS imager, hyper spectral imaging, flat field correction.

\section{INTRODUCTION}

Spectroscopy is the study of light that is emitted by or reflected from materials and its variation in energy with wavelength [1]. Hyper Spectral Imaging (HSI) is the combination of spectroscopy and traditional imaging giving a spectroscopic measurement for every pixel in the image. This typically leads to a better classification, identification and understanding of the scene [2]. A hyper spectral camera therefore contains a spectral unit next to the image sensor to map the light of different narrow bands of wavelengths on different pixels. Consequently, the amount of light energy and its intensity that falls on the image sensor is quite small compared to the traditional wide-band RGB filter in a normal imaging system. In the typical pixel structure, the electron well of the pixel is normally connected with a transistor which is in off-state while the photodiode is integrating the light. In the off-state, there exists a leakage current from drain to source of the transistor [4]. In strong light conditions, the leakage is neglected because the signal, i.e. the photon-electron generation rate, is much faster than the leakage. However, when a pixel is in a low illumination condition, as in many HSI applications, the photon-electron generation rate is in the same order of magnitude as the leakage current. In this situation, it is possible that the electrons leakage equals to the electrons generation, which builds a dynamic balance. This leads to a dynamic saturation

Manuscript received October 14, 2012; revised January 24, 2013.

Shuyang Liu and Pradip Mainali are from Katholieke Universiteit Leuven and in collaboration with Interuniversity MicroElectronics Centre (IMEC), Belgium. The corresponding author is Shuyang Liu. (e-mail: shuyang@imec.be,mainali@imec.be).

Klaas Tack and Rudy Lauwereins are with Interuniversity MicroElectronics Centre (IMEC), Belgium (e-mail: tackk@imec.be, rudy.lauwereins@imec.be). where the electrons well is not full but the collected electrons number will not increase as the exposure time increases. Even worse, the saturation level changes with light intensity as shown in Fig. 1.

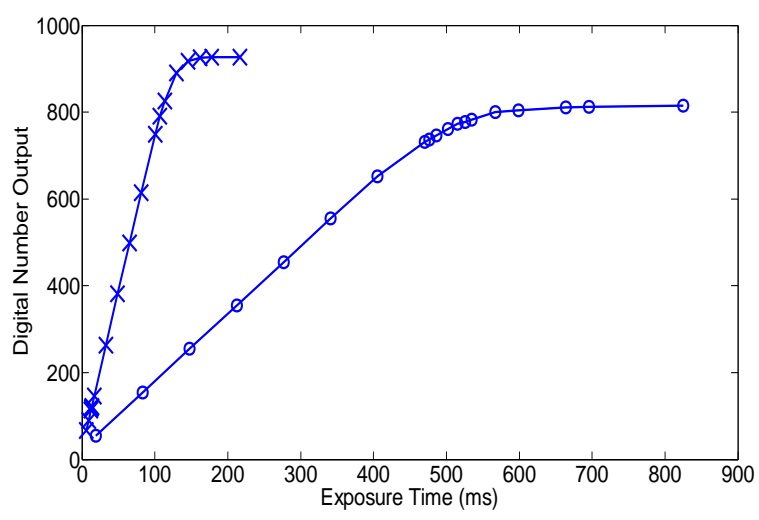

Fig. 1. Sensor response curve under two different illuminations

Therefore, for a given response, the user has no idea whether it is saturated or not because the saturation level for the corresponding illumination is unknown.

Flat Field Correction (FFC), a common method applied for removing Fixed Pattern Noise (FPN) in the imaging sensor [5], is one of the applications suffering from this dynamic saturation. Basically, the FFC uses two training images which are supposed to be linearly related to the test image to remove FPN. However, the linear relationship cannot be guaranteed between training and test images if the user has no idea about whether the training image is saturated or not.

To know the saturation level upfront, in this paper, a leakage model based on classical transistor theory is proposed and validated. The model is able to predict the saturation level and accurately describes the actual saturation level of the sensor. The model is easy to be calibrated without any extra instrument. Moreover, it also provides an easy training selection method for FFC.

The rest of this paper is organized as follows: Section II introduces the problem in state-of-the-art. The leakage principle and model are discussed in Section III. The improved FFC algorithm based on leakage model is introduced in section IV. The experiment results are shown and discussed in Section V. The conclusion and future work are included in section VI.

\section{STATE-OF-THE-ART}

A common used linear pixel response model in state-of-the-art, such as [6] is:

$$
Y=k \bullet t+d
$$


where $d$ is the dark offset, representing the response of the sensor without illumination. The parameter $k$ is the product of the light intensity, the quantum efficiency and the sensor gain The latter parameters are fixed for a given sensor; therefore the user can calculate light intensity by measuring $k$. In Fig. 2, $k$ can be interpreted as the slope of the line connected by response and dark offset. The data validity is ensured by avoiding any pixel with a maximum output, such as 1024 for a 10-bit sensor.

This model has been used many applications such as in [7] , [8].However, this is not accurate enough when the sensor is in low illumination because the transistor leakage is too big to be neglected.

Due to the dynamic saturation in low illumination, the data validity cannot be guaranteed anymore by a fixed saturation level. In Fig. 2, the measurements of a 10-bit hyper spectral sensor at different ET are shown. In traditional theory, all the responses plotted will be considered as valid because all the responses are lower than 1024. However, it is clear that the triangle data has already been saturated. For the circle data, although it is not fully saturated, it is still a good idea to avoid the non-linear data since it provides a different $\mathrm{k}$ compared with the valid data marked by pentagram. To avoid capturing the invalid data, based on the leakage model, we also develop a response model which is able to decide the validity of response without knowing the complete response curve over ET.

For the testing set selection of FFC algorithms, the data with high SNR and linearity are always appreciated. But the linearity is not able to be quantified by traditional methods without the complete response curve as the solid cure in Fig. 2. It is true that, for training purpose, the complete response function can be obtained to analysis the response linearity for each response. However, it will greatly increase the cost and efficiency of applying FFC on hyper spectral imager. For example, suppose we need 20 measurement data to build a response curve. The result is, for a 100 bands HSI imager where there are 100 pixels in each band, we need to collect 200,000 extra points in order to evaluate the linearity of each response. In this paper, thanks to the leakage model, we can tag the linearity only by each response itself without measuring extra data points. Therefore, the simplicity of the traditional FFC is maintained.

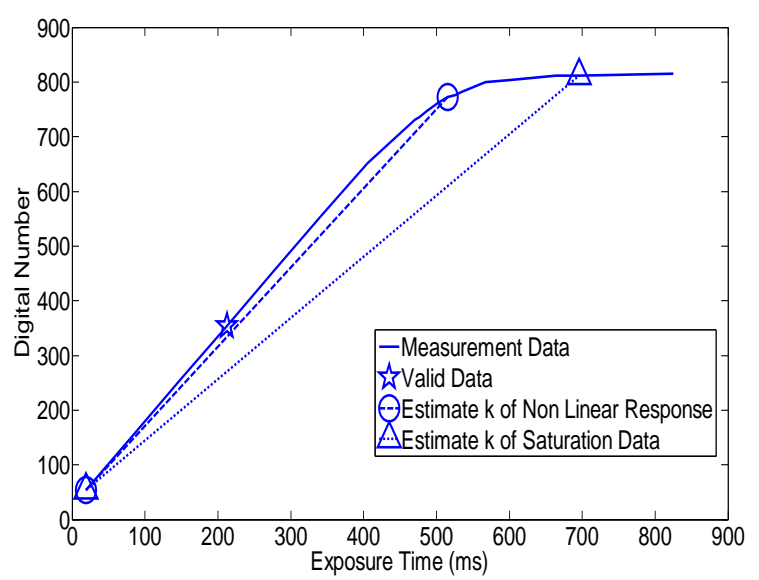

Fig. 2. Measurement of the response of a hyper spectral sensor at different exposure time

\section{LEAKAGE PRINCIPLE AND MODELING}

\section{A. Principle of Leakage in the Pixel Structure}

Part of the standard pinned, 4 Transistorpixel structure [3] is shown in Fig. 3,

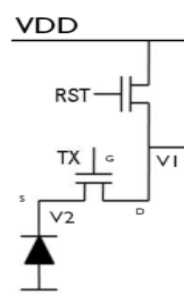

Fig. 3. Pixel structure before source follower

$\mathrm{V} 1$ is connected to a source follower, which transfers the electrons to the following read-out circuit. Before the sensor is integrating the light, the V1 is reset to VDD and V2 is at the photodiode built-in voltage, which is fixed for a specified photodiode. During the exposure time, the photodiode is receiving the photons and the generated electrons make V2 to drop, which leads to a potential difference between V1 and V2. Therefore, a leakage current will be generated from Drain to Source although the transistor is in the off state. This is called subthreshold leakage where the gate-source voltage is less than the threshold voltage [4]. The current depends exponentially on the gate-source voltage [9].

When TX is in off state, the potential $G$ is fixed. As the voltage of the source drops, both $\mathrm{V}_{\mathrm{gs}}$ and the leakage current from drain-source increase. Therefore, the leakage increases as the collected electrons increase. There are two possibilities that may happen in the exposure period. If the light intensity is so strong that, although the leakage increases as the collected electrons increase, the leakage is always lower than the speed of photon-electrons generation. In that case, the pixel will be in the real saturation if exposure time is long enough. However, if the light intensity is low, at beginning, the collected signal will also increase because the leakage is small. But at one point of the time, the leakage rate will increase till the same as the photon-electrons generation rate. Then the dynamic balance is achieved. The pixel is in the status of dynamic saturation.

\section{B. Pixel Response Model in Low Light Environment}

Because the leakage is exponential to the collected signal [9], the leakage can be represented as $a \cdot \exp (b \bullet Y)$, where $a$ and $b$ are two parameters for the leakage model and $Y$ is the output signal digital number. To include leakage inside the function, the leakage is subtracted after making (1) into differential format. Therefore, at each time we can build a differential equation

$$
d Y / d t=k-a \cdot \exp (b \cdot Y)
$$

where $k$ is the linear increasing ratio due to the light. This can be considered as the same $k$ in (1) because there is no leakage component in (1) and all the signal changing is due to the light shining at the sensor which is the signal. In (2), after the leakage is included, the $k$ is not the general response changing rate anymore as in (1) but $k$ can be interpreted as the response changing rate due to the light signal. If we integrate (2), we get 


$$
Y=\frac{1}{b} \cdot \ln \left(\frac{k \cdot e^{b \cdot k \cdot(t+c)}}{1+a \cdot e^{b \cdot k \cdot(t+c)}}\right)
$$

$c$ is a constant coming from the integration procedure. Equation (3) is the pixel response model including the leakage, which can be used to predict the saturation level. To describe the leakage influence quantitatively, another parameter is developed named leakage percentage defined as:

$$
\alpha=a \cdot e^{b \cdot Y} / k
$$

This describes how big the leakage is relatively to the input signal and helps in deciding the actual deviation from linearity, i.e. are we in saturation or not. A lower value of $\alpha$ refers to very small leakage, so the response is far away from saturation such as the pentagram data in Fig. 2 with $\alpha$ of $0.12 \%$. A higher $\alpha$ means the response has been saturated such as the triangle data in Fig. 2 with $\alpha 173.5 \%$. The ratio more than 1 does not mean the leakage is more than the signal current because the leakage will stop increasing after the dynamic saturation is achieved. A ratio more than 1 suggests the response has been saturated for quite a long time. For the response in Fig. 2, the response is saturated at around $600 \mathrm{~ms}$ and the triangle data is measured at $700 \mathrm{~ms}$. That's the reason why $\alpha$ of the triangle data is so high. For the circle data, $\alpha$ is $74.63 \%$ which means it has not been fully saturated but the leakage has already been considerably strong, the user should take care if they want to use that data close to saturation.

One more advantage of $\alpha$ is that it can be calculated easily. For a given sensor, $\mathrm{a}$ and $\mathrm{b}$ are found using $\mathrm{a}$ calibration of the sensor. The parameter $\mathrm{k}$ is approximately expressed as the $\mathrm{Y}$ divided by ET. Therefore, we can calculate $\alpha$ as long as we know the exposure time of the response, which is normally the case in practice.

To validate the response, user can decide a threshold for $\alpha$ where all the responses with $\alpha$ higher than threshold will be considered as invalid. However, the exact number of this threshold is not easy to decide. According to Fig. 2 and corresponding $\alpha$ values, a lower threshold, which refers to lower leakage, will lead to a short exposure time, which is normally linked with a lower SNR. Therefore, lower leakage and higher SNR is not easy to be achieved at the same time. User has to make a decision about the trade-off based on application. This tradeoff will be discussed in detail in section V.D.

\section{Modeling Method and Parameters Estimation}

In Equation (3), four parameters need calibration: $\mathrm{k}, \mathrm{a}, \mathrm{b}$ and $c$. The calibration procedure is as follows:

1) Calibrate $k$ : For a given light condition, several images at different ET are taken. The saturation data should be avoided. For each pixel in the sensor, we plot the response over time such as in Fig. 2. The slope of the cure corresponds to $\mathrm{k}$.

2) Calibrate $a$ and $b$ : For several different illuminations, record the saturation images and the k. For each pixel, plot the saturated digital number and $\mathrm{k}$ pairs. Because $\mathrm{k}$ equals the leakage in the dynamic saturation, we build a relationship between digital numbers versus leakage as shown in Fig. 4. The $a$ and $b$ can be obtained by interpolating the measurement data based on an exponential function.

3) Calibrate c: To keep (3) continuous, an image at short exposure time $\left(3.2^{*} 10^{-6} \mathrm{~s}\right.$ in this experiment) is also recorded. This image is employed to initialize $\mathrm{c}$ in the function.

\section{IMPROVED Two POINTS FFC ALGORITHM}

The problem of applying the traditional Two-Points FFC (TPFFC) [10]on HSI is that there are no specified rules to guarantee the training set is not saturated and linear related to the test image. Since the leakage model has been developed in the last section, this can be solved by the leakage percentage as proposed in (4). Therefore, the improved TPFFC including a new training set selection method based on leakage percentage is as follow:

1) Generate uniform light for training: The basic purpose of training images is to provide an example of the imager response while all the pixels are receiving the same amount of energy. Therefore, the illumination should be uniform over the sensor. This can be achieved by using an integration sphere for the training lamp.

2) Selecting the exposure time for training set: After the threshold of $\alpha$ is decided by user. The selected exposure time is the longest one that still make $\alpha$ lower than the threshold. The fact selecting the longest exposure time is based on the assumption that longer exposure time can provide higher SNR. However, if SNR is not increasing with exposure time, it means SNR is saturated at that exposure time. In that case, the user should lower the threshold of $\alpha$ to provide a response with less leakage since SNR is not decreased by reducing the exposure time.

3) Collecting training data: At the selected exposure time, record two responses of the imager under no illumination and under training light respectively. The two responses are referred toR(L1) and $\mathrm{R}(\mathrm{L} 2)$. To reduce noise sources such as photon shot noise, $\mathrm{R}(\mathrm{L} 1)$ and $\mathrm{R}(\mathrm{L} 2)$ can be the average result of several images which are continuously taken at the same exposure time.

4) Capture testing data: For a different light, refers to different spectrum and intensity with training light, take an image at any exposure time. This is represented by $\mathrm{R}(\mathrm{L} 3)$. Average R(L3) over the sensor, the result is the scale factor for the final correction.

5) Correct test image at L3 by using the formula:

$$
R_{n e w}=A v g(L 3) \cdot \frac{R(L 3)-R(L 1)}{R(L 2)-R(L 1)}
$$

Notice that the procedure 2 is the contribution of this paper. By doing that, the user have a way of selecting the quality of training set before really applying it on the training set. In the state-of-the-art, this TPFFC is only a basic method, not always the best. There are lots of other FFC algorithms where polynomial coefficients are used but not only the linear coefficient such as in [11]. However, all of them need a valid training set to determine the coefficients. In this paper, TPFFC is only used as an example to illustrate that the 
leakage model is not limited to TPFFC and is able to provide guidance to all FFC algorithm training set selection.

\section{EXPERIMENTS RESULTS AND DISCUSSION}

In this section, the leakage model is validated. Moreover, results of the FFC based on the different training sets are shown to demonstrate how the best training set is selected. The tradeoff among exposure time, spectrum correctness and response uniformity is discussed at the end.

\section{A. Experiment Set $U p$}

The sensor tested was a multi-spectral sensor with 8 bands. There are two different lamps from Paulmann for training and testing. They have different color temperature and therefore can provide different spectrum. By doing that, our method is proved not only working in the training illumination but also in other illuminations. Lamp A has color temperature of 2900Kand is used for training. Lamp B has color temperature of $2200 \mathrm{~K}$ is used for testing in the experiment. The uniform light is provided with integration sphere. And each training image mentioned is the average of 10 images to reduce the noise sources such as shot noise. The test image is only the raw image without any processing if not mentioned.

\section{B. Leakage Model Calibration and Response Simulation}

Before the validation, the parameters are firstly estimated for the leakage model. By using the method stated in section III.C, we measured 16 saturated digital number and $\mathrm{k}$ pairs under lamp A. The parameters $a$ and $b$ are obtained by interpolation using an exponential function and the result is shown in red in Fig. 4.

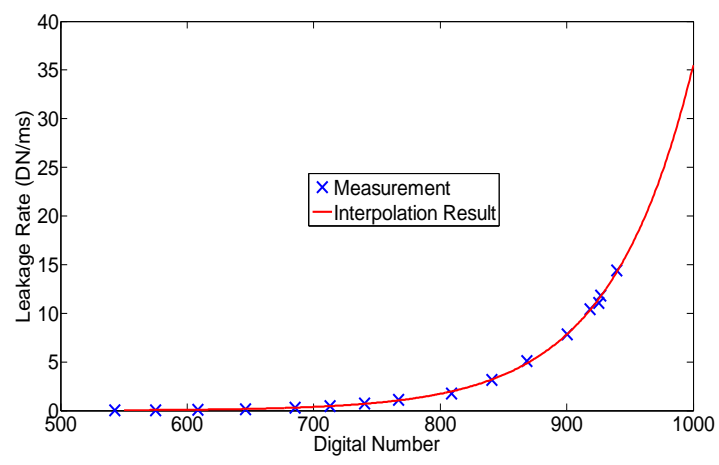

Fig. 4. Measurement and interpolation result or one pixel

After all the parameters have been determined, the sensor response can be simulated by (3). To validate the model, the simulation and measurement results under lamp B are compared as shown in Fig. 5.

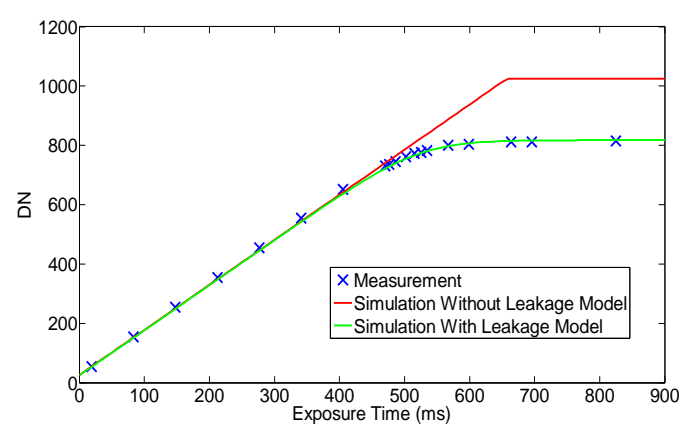

Fig. 5. Sensor response simulation and measurement

Notice the result is the average response of one entire band.

The experiment results shown in Fig. 5 illustrate the simulation including the leakage model has greatly improved the simulation accuracy, especially after $430 \mathrm{~ms}$ where the leakage started to have big influence on the response. Also, we can observe that in Fig. 5 the $\mathrm{k}$ is around 2 and saturation level is around 800 . This also coincides with the red curve in the Fig. 4 where leakage rate is also around 2 when digital number is 800 .

\section{Improved TPFFC}

In this part, our proposed method is demonstrated to be able to select the proper training set for TPFFC without taking extra measurements. The leakage percentages of all the candidate training sets are first calculated and shown in Table I. For the leakage percentage threshold, as discussed before, the user should decide it based on specified application. But we can take a $12 \%$ for an example to see how it behaves. The bold numbers represent the selected training set for TPFFC.

TABLE I: The LEAKage PeRCENTAge of All the CANDidate Training SETS

\begin{tabular}{l|lllcl}
\hline & $6.45 \mathrm{~ms}$ & $12.9 \mathrm{~ms}$ & $19.35 \mathrm{~ms}$ & $25.8 \mathrm{~ms}$ & $32.25 \mathrm{~ms}$ \\
\hline Band 1 & 0.04 & 0.11 & 0.47 & $\mathbf{5 . 1 5}$ & 37.15 \\
Band 2 & 0.01 & 0.13 & $\mathbf{1 0 . 4 0}$ & 12.92 & 66.97 \\
Band 3 & 0.01 & 0.09 & $\mathbf{1 . 3 9}$ & 17.93 & 76.44 \\
\hline
\end{tabular}

The test image is taken at $16.125 \mathrm{~ms}$ under lamp B. After applying the TPFFC using corresponding training set, the uniformity improvement is shown in Table II, where a higher number suggests a better performance of TPFFC. To get a fair uniformity evaluation of different responses level, the uniformity is scaled by the average response and defined as in (6)

$$
\text { Uniformity }=\frac{S T D(\operatorname{Img})}{\text { mean }(\operatorname{Img})} \cdot 100 \%
$$

In the Table II, we can see our selected training set is able to provide the best result of FFC among the 5 candidate training sets. Take band 1 as example, for the training set at $32.25 \mathrm{~ms}$, because the response is near to saturation with a leakage percentage of $37.15 \%$, it provides an even noisier image after FFC, represented as a negative improvement $-0.2 \%$ in Table II. For those training sets with ET shorter than $25.8 \mathrm{~ms}$, they suffer from the low SNR so that the performance of FFC is also limited. Notice that, we select the training set before we get the experiment result of Table II. In this way, the users can know the efficiency of the training set before really testing it.

TABLEII: THE UNIFORMITY IMPROVEMENT BY TwO POINTS FFC FOR DiFFERENT TRAINING IMAGES (PERCENTAGE)

\begin{tabular}{l|lllll}
\hline & $6.45 \mathrm{~ms}$ & $12.9 \mathrm{~ms}$ & $19.35 \mathrm{~ms}$ & $25.8 \mathrm{~ms}$ & $32.25 \mathrm{~ms}$ \\
\hline Band 1 & 0.03 & 0.14 & 0.16 & $\mathbf{0 . 1 7}$ & -0.20 \\
Band 2 & 0.03 & 0.15 & $\mathbf{0 . 1 8}$ & 0.13 & -0.90 \\
Band 3 & 0.04 & 0.11 & $\mathbf{0 . 1 6}$ & 0.03 & -1.16 \\
\hline
\end{tabular}

\section{Spectrum Correctness}

In this part, the leakage percentage is demonstrated to be able to avoid spectrum deformity. The principle for the 
spectrum comparison is that the same spectral sensor should provide same spectrum for the same light at different exposure time. For those spectrums having the deformed shapes, the leakage percentage is able to detect their invalidity.

We collect 11 spectrums of lamp B by 11 different exposure time sand each spectrum is normalized by maximum before they are plotted in Fig. 6.The threshold is $12 \%$ as before. The invalid data is shown in dot in Fig. 6.

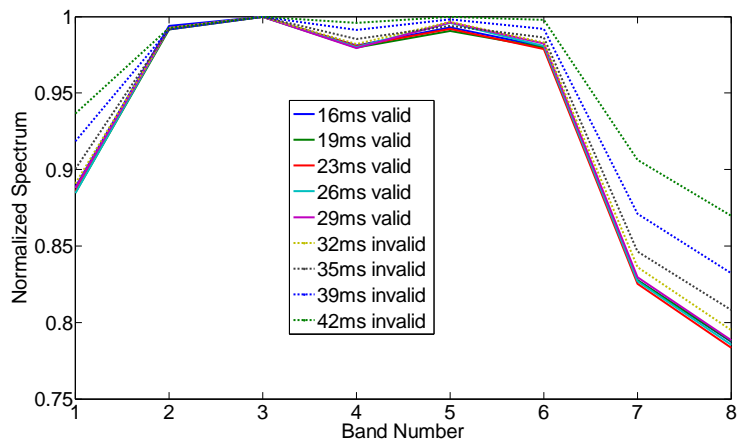

Fig. 6. Spectrum of lamp B at different exposure time

The result shows the spectrum tends to be deformed while the ET increasing more than $29 \mathrm{~ms}$ because some of the bands are started to be saturated. In Contrast, the valid spectrum is sharing more similar shape than the others, which means the deformed spectrums such as those with exposure time more than $29 \mathrm{~ms}$ are avoided.

It is not difficult to explain how the spectrums are changing their shapes after saturation. Take band 1 and 2 as an example, after $32 \mathrm{~ms}$, the response of band 1 increase from 0.9 to 0.94 while the response of band 2 doesn't change a lot due to saturation, this leads to the spectrum deformity in the region of band 1 and band 2 . This also explains why the responses at the non-saturated bands tend to increase after one band is saturated.

Now the question will be whether the leakage percentage threshold can be globally optimized. Together with the results of this section, a general trade off among SNR, Spectrum Correctness (SC) and Response Uniformity (RU) can be built. SC and RU are in the same side whereas the SNR is in the other side. While SNR is too low, both SC and RU are not good. In the other hand, if the SC and RU are too bad, no matter how high the SNR is, the result is still invalid. For those cases which are between these two extreme conditions, a case-by-case method should be appreciated. For each application, there should be a weight to evaluate the importance of SNR, SC and RU. Only after those are known, a local optimized threshold for leakage percentage is possible for the specific application.

\section{CONCLUSION}

A leakage model based on the standard CMOS imaging pixel structure is proposed including the calibration methods. The model is able to provide a way for user to tag the validity of a response. The results have shown this model is helpful to improve simulation accuracy and avoid the spectrum deformity. Moreover, the model is also demonstrated to be able to provide guidance to the training set selection of TPFFC algorithm. However, the exact number of the leakage percentage threshold should be application dependent under a decent consideration including the trade off among SNR, response uniformity and spectrum correctness.

In the future work, more time will be spent on comparing the object reflectance spectrum with a benchmark system to further validate the performance of the leakage model. Moreover, an improved FFC algorithm is also appreciated which can improve the uniformity while keeping the spectrum unchanged for each pixel.

\section{ACKNOWLEDGMENT}

The authors would like to thank Mr. Bart Masschelein from IMEC for his effort on the hyper-spectral lab maintenance where the authors have finished the experiment.

\section{REFERENCES}

[1] R. N. Clark and T. L. Roush, "Reflectance spectroscopy: Quantitative analysis techniques for remote sensing applications," Journal of Geophysical Research, pp. 6329-6340, 1984.

[2] P. Shippert, "Why use hyperspectral imagery," Photogarammetric Enginneering and Remote Sensing 70, 2004, pp. 377-380.

[3] A. Krymski, N. Khaliullin, and H. Rhodes, "A 2 e- noise 1.3 Megapixel CMOS sensor," IEEE Workshop on CCDs and Advanced Image Sensors, 2003.

[4] J. Kao, S. Narendra, and A. Chandrakasan, "Subthreshold leakage modeling and reduction techniques," in Proceedings of the 2002 IEEE/ACM international conference on Computer-aided design 2002, pp. 141-148.

[5] J. A. Seibert, J. M. Boone, and K. K. Lindfors, "Flat-field correction technique for digital detectors," Medical Imaging 1998: Physics of Medical Imaging, 1998, pp. 348-354.

[6] J. Farrell, M. Okincha, and M. Parmar, "Sensor calibration and simulation," in Proc. SPIE Int. Soc. Opt, 2008, vol. 6817.

[7] T. Chen, "Digital camera system simulation and applications," Ph.D. dissertation, Dept. Elect. Eng., Stanford Univ., USA, 1993.

[8] P. L. Vora, J. E. Farrell, and J. D. Tietz, "Image capture: simulation of sensor responses from hyperspectral images," IEEE Transaction on Image Processing, 2001, pp. $307-316$.

[9] Y. P. Tsividis, "Operation and Modeling of the MOSTransistor," McGraw-Hill, New-York, 1987, ch. 3.

[10] L. M. Webb and D. A. Auger, "Use of flat-field correction and adaptive linear interpolation to improve mammography image quality," in Proceedings of the 22nd Annual Symposium of the Pattern Recognition Association of South Africa, Emerald Casino, 2011, pp. 150-155.

[11] C. W. Timothy and C. R. Shaddix, "Simultaneous correction of flat field and nonlinearity response of intensified charge-coupled devices," Review of Scientific Instruments, 2007, pp. 123702.1-123702.6.

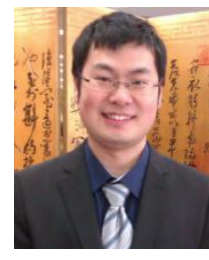

Shuyang Liu obtained his bachelor of science from Nankai University, China in 2008 and master in color information jointly from University of Sainte-Etienne, France and University of Eastern Finland, Finland in 2010 under the scholarship of European Erasmus Mundus Programme. Now he is a Ph.D. student at Katholieke Universiteit Leuven in collaboration with IMEC, Belgium. His research includes spectral imaging system simulation and application co-design.

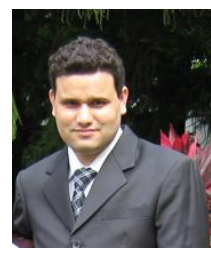

Pradip Mainali obtained his Bachelor of Engineering from National Institute of Technology, Surat, India in 2002 and Master of Technology Design in Embedded Systems jointly awarded by National University of Singapore, Singapore and Technical University of Eindhoven, Netherlands in 2006. From 2005 to 2009, he worked as an embedded software engineer in Motorola. He is currently a Ph.D. student at Katholieke Universiteit Leuven and in collaboration with IMEC, Belgium. His research interest includes computer vision and machine learning. 


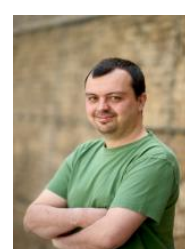

Klaas Tack received the engineering degree and a Ph.D. in electrical engineering from the Katholieke Universiteit Leuven in 2001 and 2006 respectively. He is currently a senior researcher in the integrated imaging team at imec, where he is involved in the development of a compact hyperspectral camera.

Rudy Lauwereins is vice president of imec, which performs world-leading research and delivers industry-relevant technology solutions through global partnerships in nano-electronics, ICT, healthcare and energy. He is responsible for imec's Smart Systems Technology Office, covering energy efficient green radios, vision systems, (bio)medical and lifestyle electronics as well as wireless autonomous transducer systems and large area organic electronics. He is also a part-time Full Professor at the Katholieke Universiteit Leuven, Belgium, where he teaches Computer Architectures in the Master of Science in Electrotechnical Engineering program. Before joining imec in 2001, he held a tenure Professorship in the Faculty of Engineering at the Katholieke Universiteit
Leuven since 1993. He had obtained a Ph.D. in Electrical Engineering in 1989. Rudy Lauwereins served in numerous international program committees and organizational committees, and gave many invited and keynote speeches. He was the general chair of the DATE conference (Design, design Automation and Test in Europe) in 2007. He is a senior member of the IEEE. Professor Lauwereins has authored and co-authored more than 350 publications in international journals, books and conference proceedings. 